\title{
Floristic composition and structure of an upper montane cloud forest in the Serra da Mantiqueira Mountain Range of Brazil
}

\author{
Patrícia Vieira Pompeu ${ }^{1,3}$, Marco Aurélio Leite Fontes ${ }^{1}$, Rubens Manoel dos Santos ${ }^{1}$, Paulo Oswaldo Garcia ${ }^{1}$, \\ Talita Alves Batista ${ }^{1}$, Warley Augusto Caldas Carvalho ${ }^{1}$ and Ary Teixeira de Oliveira Filho ${ }^{2}$
}

Received: June 23, 2013. Accepted: April 16, 2014

\begin{abstract}
We analyzed the community structure of an upper montane cloud forest (elevation, $1900 \mathrm{~m}$ ) in the Serra da Mantiqueira Mountain Range, in the state of Minas Gerais, Brazil. Our objective was to determine the comparative tree species richness and floristic diversity within this forest, which is at one of the higher elevations in the range, in relation to surrounding forests that are at lower elevations, adjusting for elevational gradients. Within 15 permanent plots $(40 \times 10 \mathrm{~m}$ each), we tagged all tree individuals with a diameter at breast height $\geq 5 \mathrm{~cm}$, registering their height and diameter. To compare the study area with neighboring cloud forests, we used the Sørensen similarity coefficient and phytosociological parameters. We sampled 1250 individuals distributed among 89 species, 55 genera, and 34 families. Canonical correspondence analysis revealed no gradients related to the vegetation or soil. We found that tree species richness and diversity were high in the study area. There was structural and floristic heterogeneity among the communities evaluated, underscoring the importance of conservation of these high-elevation ecosystems, which are so unique and irreplaceable.
\end{abstract}

Key words: Upper montane cloud forest, mountain forest, phytosociology, tree community, Atlantic Forest.

\section{Introduction}

The Serra da Mantiqueira Mountain Range is part of the Atlantic Forest Biome, which encompasses various types of forest formations. Cloud forests constitute a type of rain forest that occurs at higher elevations and is shrouded in low-level cloud cover for most of the year (Webster 1995). In the Serra da Mantiqueira, cloud forests typically occur above $1100 \mathrm{~m}$. Neotropical cloud forests are among the rarest and most threatened ecosystems in the world (Bruijnzeel et al. 2010), collectively occupying an estimated area of only approximately $215,000 \mathrm{~km}^{2}$, which corresponds to only $1.4 \%$ of all tropical forests and $6.6 \%$ of all montane tropical forests (Scatena et al. 2010). In Brazil, cloud forests within the southern and southeastern segments of the Atlantic Forest Biome form a distinct, albeit poorly studied, floristic and phytogeographic unit (Bertoncello et al. 2011). At the higher elevations of the Serra da Mantiqueira, Meirelles et al. (2008) and França \& Stehmann (2004) conducted tree community surveys at $1820-1940 \mathrm{~m}$ and at $1900 \mathrm{~m}$, respectively. Nevertheless, the Atlantic Forest is considered a global priority area for the conservation of cloud forests (Bruijnzeel et al. 2010). Therefore, this study aimed to expand the knowledge of cloud forest ecosystems in Brazil, describing and analyzing the community structure of an upper montane cloud forest within the Atlantic Forest Biome, in the Serra da Mantiqueira.

\section{Material and Methods}

\section{Study area}

The study site is within the High Montane Private Nature Reserve (22 $22^{\prime} 39^{\prime \prime} S$; $44^{\circ} 48^{\prime} 55^{\prime \prime} \mathrm{W}$ ), located in the county of Itamonte, within the state of Minas Gerais, Brazil. The reserve comprises 672 ha of continuous forest and montane grassland, the latter restricted to the highest elevations (2100-2400 m). The area ranks eighth among the most irreplaceable ecosystems in the world in terms of the diversity of vertebrate species, which are threatened with extinction, and there have been urgent calls for additional measures to ensure its effective management and conservation (Le-Saout et al. 2013). The study area has a history of complex disturbance, with fewer disturbances at the upper elevations. Currently, the area is strictly protected and has been incorporated into the Serra da Mantiqueira

\footnotetext{
${ }^{1}$ Universidade Federal de Lavras, Departamento de Ciências Florestais, CP 3037, Lavras, MG, CEP 37200-000, Brazil

${ }^{2}$ Universidade Federal de Minas Gerais, Departamento de Botânica, Pampulha, Belo Horizonte, MG, CEP 31270-901, Brazil

${ }^{3}$ Author for correspondence: patpompeu@hotmail.com
} 
Environmentally Protected Area. The study site, which is at an elevation of $1900 \mathrm{~m}$, is $15 \mathrm{~km}$ from the entrance to Itatiaia National Park (near the Serra Fina Mountain Range). This area is part of the Atlantic Forest Biome, and the predominant type of vegetation formation is dense high montane rain forest (IBGE 2012). The area can also be classified as tropical broadleaf evergreen upper montane cloud forest (Oliveira-Filho 2009) or simply upper montane cloud forest (Bruijnzeel \& Hamilton 2000; Bruijnzeel 2001; Scatena et al. 2010). We observed that, in comparison with the surrounding areas (at lower elevations), the study site presented greater epiphytism, with more mosses on tree trunks and in tree canopies, smaller leaf sizes and a greater abundance of vines, as well as bamboos (which do not occur at the lower elevations).

According to the Köppen classification system, the climate of the region under study is type Cwb, characterized by humid temperate with dry winters, the average temperature in the warmest and coolest months being < $17.3^{\circ} \mathrm{C}$ and $>12.7^{\circ} \mathrm{C}$, respectively (Sá Júnior et al. 2012). The historic average annual rainfall is $1749 \mathrm{~mm}$ (Pane \& Pereira 2005). Geologically, the study area is part of Paraíba do Sul Complex, covering a lithologic group comprising mainly gneisses and migmatites, which corresponds to an environment with extremely strong relief, with crests sectioned at the higher elevations due to faulting (Pane \& Pereira 2005). The resulting soils are Inceptisols (Pane \& Pereira 2005).

\section{Sampling}

We sampled the tree community in 15 permanent plots $(40 \times 10 \mathrm{~m}$ each; total area, $0.6 \mathrm{ha})$, distributed systematically at regular intervals, $15 \mathrm{~m}$ apart, all at an elevation of $1900 \mathrm{~m}$. Using numbered aluminum tags, we tagged all trees with a diameter $\geq 5 \mathrm{~cm}$ with. We also measured the circumference and height of those individuals using a tape measure and a graduated pole, respectively. The reproductive material collected was processed following the usual herbarium techniques and deposited in the following herbaria: ESAL, UEC, RB; which acronyms are in accordance with the Index Herbariorum (Thiers continuously updated) plus the Herbarium at the State University of Montes Claros. For the classification of specimens down to the family level, we followed the Angiosperm Phylogeny Group III guidelines (APG III 2009).

Texture and chemical analyses of one composite soil sample from each plot, sampled in the center and vertices, were conducted at the Soil Analysis Laboratory at the Federal University of Lavras following the Brazilian Agency for Agricultural Research protocol (EMBRAPA 1997). The following soil variables were measured: $\mathrm{pH}$; levels of $\mathrm{P}, \mathrm{K}, \mathrm{Ca}$, $\mathrm{Mg}, \mathrm{Al}$, and $\mathrm{H}+\mathrm{Al}$; sum of bases; base saturation; aluminum saturation; cation exchange capacity; cation exchange capacity at $\mathrm{pH}$ 7.0; remaining phosphorus; organic matter; and sand, silt, and clay fractions.

\section{Data analysis}

To describe the horizontal structure of the tree community, we calculated the classical phytosociological parameters for each species (Mueller-Dombois \& Ellenberg 1974): absolute and relative density; absolute and relative dominance; absolute and relative frequency; and importance value, which is the sum of the aforementioned relative values. To evaluate species diversity, we employed the Shannon diversity index and Pielou's evenness index (Brower \& Zar 1997), applying a rarefaction curve, based on species richness versus the number of individuals, in the program PCORD, version 5.1 (Mccune \& Mefford 2006). For the species with the highest importance values, we drew statistical comparisons for abundance and basal area using one-way ANOVA followed by Tukey's test (Zar 2010), which allowed us to recognize patterns of structural organization within populations. Data normality were tested, and parameters were transformed by square root functions (for abundance) or log transformed (for basal area) when necessary.

The correlations between soil variables and species abundance were analyzed by canonical correspondence analysis (CCA), again with PC-ORD. The CCA was performed to evaluate floristic patterns correlated with soil variables. From the abundance data of the 23 species represented by five or more individuals, we constructed a species composition matrix in which values were log transformed to compensate for deviations caused by outliers (ter Braak 1995). To create the soil data matrix, we performed a preliminary CCA, eliminating correlated environmental variables or those that correlated only weakly with the ordination axes. Therefore, the final CCA was calculated using the following variables: sand, silt, and clay fractions; $\mathrm{pH}, \mathrm{P}, \mathrm{K}, \mathrm{Ca}$, and $\mathrm{Al}$; sum of bases; cation exchange capacity at $\mathrm{pH}$ 7.0; organic matter; and remaining phosphorus. We used the Sørensen similarity coefficient (Brower \& Zar 1984) and phytosociological parameters in order to compare the study area with other cloud forests studied in the Serra da Mantiqueira: at Poços de Caldas (Costa et al. 2011); at Camanducaia (França \& Stehmann 2004); at Monte Verde (Meireles et al. 2008); and at Bocaina de Minas (Carvalho et al. 2005).

\section{Results}

We sampled 1250 individuals distributed among 89 species, 55 genera, and 34 families (Tab. 1). The families with highest species richness were Myrtaceae, with 20 species; Melastomataceae, with nine; Lauraceae, with six; Aquifoliaceae, with four; Myrsinaceae, with four; and Rubiaceae, with four. Those six families accounted for $53 \%$ of all of the species sampled. There were 19 families that were represented by a single species. The genera with highest species richness (collectively accounting for $42 \%$ of the species sampled) were Miconia and Myrcia (six species 
Patrícia Vieira Pompeu, Marco Aurélio Leite Fontes, Rubens Manoel dos Santos, Paulo Oswaldo Garcia,

Talita Alves Batista, Warley Augusto Caldas Carvalho and Ary Teixeira de Oliveira Filho

Table 1. List of tree species sampled in 0.6 ha of an upper montane cloud forest at $1900 \mathrm{~m}$ elevation in the Serra da Mantiqueira Mountain Range, Itamonte County, in the state of Minas Gerais, Brazil.

\begin{tabular}{|c|c|c|c|c|c|c|}
\hline Species & $\mathrm{CN}$ & $\mathrm{n}$ & $\mathrm{AD}$ & ADo & $\mathrm{AF}$ & $\mathrm{IV}^{*}$ \\
\hline Roupala rhombifolia Mart. ex Meisn. & TAB 377 & 94 & 156.7 & 5.043 & 86.7 & 26.5 \\
\hline Myrsine umbellata Mart. & ТАВ 149 & 93 & 155.0 & 3.345 & 86.7 & 21.3 \\
\hline Pimenta pseudocaryophyllus (Gomes) Landrum & TAB 303 & 84 & 140.0 & 2.654 & 93.3 & 18.8 \\
\hline Myrceugenia miersiana (Gardner) D.Legrand \& Kausel & - & 90 & 150.0 & 2.067 & 100.0 & 17.7 \\
\hline Myrcia splendens (Sw.) DC. & TAB 276 & 89 & 148.3 & 1.881 & 86.7 & 16.5 \\
\hline Prunus myrtifolia (L.) Urb. & ТАВ 277 & 88 & 146.7 & 1.759 & 93.3 & 16.4 \\
\hline Ilex brevicuspis Reissek & TAB 343 & 58 & 96.7 & 1.469 & 93.3 & 13.1 \\
\hline Miconia pusilliflora (DC.) Triana & TAB 271 & 83 & 138.3 & 0.749 & 80.0 & 12.3 \\
\hline Calyptranthes brasiliensis Spreng. & - & 55 & 91.7 & 1.615 & 66.7 & 12.1 \\
\hline Cabralea canjerana (Vell.) Mart. & TAB 323 & 48 & 80.0 & 1.016 & 86.7 & 10.6 \\
\hline Psychotria vellosiana Benth. & ТАВ 185 & 56 & 93.3 & 0.590 & 80.0 & 9.7 \\
\hline Lamanonia ternata Vell. & ТАВ 172 & 26 & 43.3 & 1.112 & 66.7 & 8.3 \\
\hline Siphoneugena widgreniana O.Berg & TAB 355 & 32 & 53.3 & 0.585 & 73.3 & 7.5 \\
\hline Miconia sellowiana Naudin & TAB 146 & 24 & 40.0 & 0.474 & 60.0 & 5.9 \\
\hline Eugenia florida DC. & - & 24 & 40.0 & 0.370 & 53.3 & 5.3 \\
\hline Clethra scabra Pers. & TAB 168 & 10 & 16.7 & 0.671 & 40.0 & 4.5 \\
\hline Ilex paraguariensis A.St.-Hil. & ТАВ 344 & 12 & 20.0 & 0.423 & 40.0 & 4.0 \\
\hline Cryptocarya aschersoniana Mez & TAB 359 & 13 & 21.7 & 0.447 & 33.3 & 3.8 \\
\hline Croton piptocalyx Müll.Arg. & TAB 397 & 7 & 11.7 & 0.497 & 40.0 & 3.8 \\
\hline Piptocarpha macropoda Baker & ТАВ 414 & 10 & 16.7 & 0.208 & 53.3 & 3.7 \\
\hline Siphoneugena densiflora O.Berg & TAB 354 & 16 & 26.7 & 0.358 & 26.7 & 3.5 \\
\hline Tibouchina estrellensis (Raddi) Cogn. & TAB 206 & 19 & 31.7 & 0.257 & 26.7 & 3.4 \\
\hline Guatteria australis A.St.-Hil. & ТАВ 194 & 9 & 15.0 & 0.291 & 40.0 & 3.3 \\
\hline Symplocos insignis Brand & TAB 489 & 11 & 18.3 & 0.257 & 33.3 & 3.1 \\
\hline Byrsonima laxiflora Griseb. & TAB 338 & 5 & 8.3 & 0.271 & 33.3 & 2.6 \\
\hline Eugenia dodonaeifolia Cambess. & - & 8 & 13.3 & 0.369 & 20.0 & 2.6 \\
\hline Cinnamomum triplinerve (Ruiz \& Pav.) Kosterm. & ТАВ 471 & 8 & 13.3 & 0.275 & 26.7 & 2.6 \\
\hline Drimys brasiliensis Miers & ТАВ 491 & 11 & 18.3 & 0.253 & 20.0 & 2.5 \\
\hline Miconia castaneiflora Naudin & TAB 231 & 9 & 15.0 & 0.062 & 33.3 & 2.3 \\
\hline Solanum pseudoquina A.St.-Hil. & ТАВ 268 & 4 & 6.7 & 0.259 & 26.7 & 2.2 \\
\hline Rudgea jasminoides (Cham.) Müll.Arg. & TAB 334 & 10 & 16.7 & 0.075 & 26.7 & 2.2 \\
\hline Cestrum axillare Vell. & - & 5 & 8.3 & 0.105 & 33.3 & 2.1 \\
\hline Myrcia venulosa DC. & TAB 353 & 6 & 10.0 & 0.064 & 33.3 & 2.1 \\
\hline Maytenus gonoclada Mart. & TAB 340 & 6 & 10.0 & 0.246 & 20.0 & 2.1 \\
\hline Ouratea semiserrata (Mart. \& Nees) Engl. & - & 10 & 16.7 & 0.077 & 20.0 & 1.9 \\
\hline Citronella paniculata (Mart.) R.A.Howard & TAB 339 & 4 & 6.7 & 0.137 & 26.7 & 1.9 \\
\hline Myrceugenia bracteosa (DC.) D.Legrand \& Kausel & ТАВ 413 & 8 & 13.3 & 0.308 & 6.7 & 1.9 \\
\hline Maytenus evonymoides Reissek & ТАВ 267 & 5 & 8.3 & 0.069 & 26.7 & 1.7 \\
\hline Daphnopsis brasiliensis Mart. \& Zucc. & TAB 336 & 6 & 10.0 & 0.074 & 20.0 & 1.6 \\
\hline Cyathea phalerata Mart. & TAB 356 & 7 & 11.7 & 0.178 & 6.7 & 1.4 \\
\hline
\end{tabular}


Floristic composition and structure of an upper montane cloud forest in the Serra da Mantiqueira Mountain Range of Brazil

Table 1. Continuation.

\begin{tabular}{|c|c|c|c|c|c|c|}
\hline Species & $\mathrm{CN}$ & $\mathrm{n}$ & $\mathrm{AD}$ & ADo & $\mathrm{AF}$ & $\mathrm{IV}^{*}$ \\
\hline Miconia latecrenata (DC.) Naudin & - & 10 & 16.7 & 0.074 & 6.7 & 1.3 \\
\hline Eugenia handroana D.Legrand & ТAB 236 & 3 & 5.0 & 0.069 & 20.0 & 1.3 \\
\hline Symplocos celastrinea Mart. ex Miq. & TAB 330 & 3 & 5.0 & 0.162 & 13.3 & 1.3 \\
\hline Psidium rufum DC. & - & 4 & 6.7 & 0.088 & 13.3 & 1.2 \\
\hline Mollinedia clavigera Tul. & TAB 326 & 2 & 3.3 & 0.116 & 13.3 & 1.1 \\
\hline Piptocarpha axillaris (Less.) Baker & TAB 342 & 2 & 3.3 & 0.107 & 13.3 & 1.1 \\
\hline Myrcia multiflora (Lam.) DC. & - & 3 & 5.0 & 0.064 & 13.3 & 1.0 \\
\hline Euplassa organensis (Gardner) I.M.Johnst. & - & 3 & 5.0 & 0.028 & 13.3 & 0.9 \\
\hline Calyptranthes clusiifolia O.Berg & - & 2 & 3.3 & 0.054 & 13.3 & 0.9 \\
\hline Dasyphyllum brasiliense (Spreng.) Cabrera & TAB 341 & 1 & 1.7 & 0.173 & 6.7 & 0.9 \\
\hline Leandra quinquedentata (DC.) Cogn. & TAB 320 & 2 & 3.3 & 0.051 & 13.3 & 0.9 \\
\hline Ocotea minarum (Nees \& Mart.) Mez & TAB 357 & 2 & 3.3 & 0.036 & 13.3 & 0.8 \\
\hline Myrcia retorta Cambess. & ТAB 352 & 3 & 5.0 & 0.102 & 6.7 & 0.8 \\
\hline Daphnopsis fasciculata (Meisn.) Nevling & - & 2 & 3.3 & 0.022 & 13.3 & 0.8 \\
\hline Ocotea corymbosa (Meisn.) Mez & - & 2 & 3.3 & 0.020 & 13.3 & 0.8 \\
\hline Ilex sapotifolia Reissek & TAB 345 & 2 & 3.3 & 0.015 & 13.3 & 0.8 \\
\hline Myrsine lineata (Mez) Imkhan. & - & 4 & 6.7 & 0.044 & 6.7 & 0.7 \\
\hline Guapira opposita (Vell.) Reitz & - & 1 & 1.7 & 0.113 & 6.7 & 0.7 \\
\hline Myrsine coriacea (Sw.) R.Br. ex Roem. \& Schult. & TAB 243 & 1 & 1.7 & 0.078 & 6.7 & 0.6 \\
\hline Agarista eucalyptoides (Cham. \& Schltdl.) G.Don & TAB 322 & 1 & 1.7 & 0.071 & 6.7 & 0.6 \\
\hline Calyptranthes widgreniana O.Berg & ТАВ 349 & 2 & 3.3 & 0.040 & 6.7 & 0.6 \\
\hline Zanthoxylum fagara (L.) Sarg. & - & 1 & 1.7 & 0.061 & 6.7 & 0.6 \\
\hline Dictyoloma vandellianum A.Juss. & TAB 284 & 1 & 1.7 & 0.050 & 6.7 & 0.5 \\
\hline Roupala montana Aubl. & TAB 173 & 1 & 1.7 & 0.047 & 6.7 & 0.5 \\
\hline Heisteria silvianii Schwacke & - & 1 & 1.7 & 0.046 & 6.7 & 0.5 \\
\hline Miconia corallina Spring & TAB 239 & 2 & 3.3 & 0.015 & 6.7 & 0.5 \\
\hline Ocotea diospyrifolia (Meisn.) Mez & ТAB 358 & 2 & 3.3 & 0.012 & 6.7 & 0.5 \\
\hline Myrcia guianensis (Aubl.) DC. & TAB 148 & 2 & 3.3 & 0.011 & 6.7 & 0.5 \\
\hline Sloanea hirsuta (Schott) Planch. ex Benth. & - & 2 & 3.3 & 0.010 & 6.7 & 0.5 \\
\hline Symplocos falcata Brand & ТАВ 153 & 1 & 1.7 & 0.034 & 6.7 & 0.5 \\
\hline Agonandra excelsa Griseb. & - & 1 & 1.7 & 0.029 & 6.7 & 0.5 \\
\hline Vitex megapotamica (Spreng.) Moldenke & - & 1 & 1.7 & 0.027 & 6.7 & 0.4 \\
\hline Myrcia pulchra (O.Berg) Kiaersk. & ТAB 152 & 1 & 1.7 & 0.023 & 6.7 & 0.4 \\
\hline Ilex affinis Gardner & - & 1 & 1.7 & 0.019 & 6.7 & 0.4 \\
\hline Daphnopsis coriacea Taub. & - & 1 & 1.7 & 0.017 & 6.7 & 0.4 \\
\hline Cordiera sessilis (Vell.) Kuntze & ТAB 324 & 1 & 1.7 & 0.015 & 6.7 & 0.4 \\
\hline Miconia splendens (Sw.) Griseb. & - & 1 & 1.7 & 0.011 & 6.7 & 0.4 \\
\hline Aegiphila integrifolia (Jacq.) B.D.Jackson & - & 1 & 1.7 & 0.009 & 6.7 & 0.4 \\
\hline Blepharocalyx salicifolius (Kunth) O.Berg & TAB 348 & 1 & 1.7 & 0.009 & 6.7 & 0.4 \\
\hline Schefflera calva (Cham.) Frodin \& Fiaschi & - & 1 & 1.7 & 0.009 & 6.7 & 0.4 \\
\hline Annona cacans Warm. & TAB 347 & 1 & 1.7 & 0.009 & 6.7 & 0.4 \\
\hline
\end{tabular}


Patrícia Vieira Pompeu, Marco Aurélio Leite Fontes, Rubens Manoel dos Santos, Paulo Oswaldo Garcia,

Talita Alves Batista, Warley Augusto Caldas Carvalho and Ary Teixeira de Oliveira Filho

Table 1. Continuation.

\begin{tabular}{|c|c|c|c|c|c|c|}
\hline Species & $\mathrm{CN}$ & $\mathrm{n}$ & $\mathrm{AD}$ & ADo & $\mathrm{AF}$ & $\mathrm{IV}^{*}$ \\
\hline Aiouea saligna Meisn. & - & 1 & 1.7 & 0.008 & 6.7 & 0.4 \\
\hline Maytenus salicifolia Reissek & - & 1 & 1.7 & 0.006 & 6.7 & 0.4 \\
\hline Marlierea excoriata Mart. & - & 1 & 1.7 & 0.005 & 6.7 & 0.4 \\
\hline Leandra aurea (Cham.) Cogn. & TAB 237 & 1 & 1.7 & 0.005 & 6.7 & 0.4 \\
\hline Meliosma sellowii Urb. & - & 1 & 1.7 & 0.004 & 6.7 & 0.4 \\
\hline Myrsine gardneriana A.DC. & TAB 434 & 1 & 1.7 & 0.004 & 6.7 & 0.4 \\
\hline Euplassa legalis (Vell.) I.M.Johnst. & - & 1 & 1.7 & 0.004 & 6.7 & 0.4 \\
\hline Rhamnus sphaerosperma Sw. & TAB 332 & 1 & 1.7 & 0.004 & 6.7 & 0.4 \\
\hline Total & & 1250 & 2083 & 33.0 & 2340 & 300 \\
\hline
\end{tabular}

$\mathrm{CN}$ - collector number; $\mathrm{n}$ - number of individuals sampled; $\mathrm{AD}$ - absolute density (ind./ha); $\mathrm{ADo}$ - absolute dominance ( $\left.\mathrm{m}^{2} / \mathrm{ha}\right) ; \mathrm{AF}-\mathrm{absolute}$ frequency (\%); IV - importance value $(\%)$.

* Species are listed in decreasing order by IV.

each); Ilex and Myrsine (four species each); and Calyptranthes, Daphnopsis, Maytenus, Symplocos, Eugenia, and Ocotea (three species each). Abundance ( $\mathrm{n}$ of individuals) was greatest in the family Myrtaceae $(\mathrm{n}=434)$, which was followed by Melastomataceae $(\mathrm{n}=151)$, Myrsinaceae $(\mathrm{n}=$ 99), Proteaceae $(n=99)$, and Rosaceae $(n=88)$, those five families accounting for $70 \%$ of the individuals identified.

Among the tree species with the highest VIs in the sampling area, we found no significant differences in terms of abundance $\left(\mathrm{F}_{0.05,5.84}=0.05 ; p=0.99\right)$. However, Roupala rhombifolia showed a greater basal area than did Myrceugenia miersiana $(\mathrm{q}=4.15, p=0.04)$, Myrcia splendens $(\mathrm{q}$ $=4.4, p=0.02)$, and Prunus myrtifolia $(\mathrm{q}=4.5, p=0.02)$, resulting in significant dissimilarities among basal areas of the main populations of the tree component $\left(\mathrm{F}_{0.05,5.84}=3.09\right.$; $p=0.01$ ).

The tree community had an absolute density of 2083 ind.ha $\mathrm{h}^{-1}$ and absolute dominance of $33 \mathrm{~m}^{2} \cdot \mathrm{ha}^{-1}$. Among the individuals sampled, the mean height was $10.4 \mathrm{~m}$ and the mean diameter was $12.14 \mathrm{~cm}$. The Shannon diversity index was 3.49, and Pielou's evenness index was 0.78 . The rarefaction curve (Fig. 1) showed that most species were sampled, because the curve tended to reach an asymptote.

The results of the soil texture and chemical analyses of the 15 plots sampled are shown in Tab. 2. The eigenvalues for the first three axes of the CCA were low $(0.189 ; 0.109$; 0.103 respectively), indicating low species turnover in the sampled area (<0.5; sensu ter Braak 1995). In addition, the structural organization of the tree community did not correlate significantly with any of the soil attributes $(P=$ 0.2953), underscoring the homogeneity of the tree community in the cloud forest under study.

The comparisons among Itamonte and the other cloud forests in the Serra da Mantiqueira, in terms of structure, diversity, species richness, and most important species, are shown in Tab. 3. There were variations in basal area and abundance among the forests evaluated, basal area being greatest in Camanducaia and density being greatest in Monte Verde, whereas both were lowest in Poços de Caldas. The Sørensen similarity index indicated that there was little similarity, in terms of species composition, among the areas, the index being highest for the pairs Itamonte-Poços de Caldas (0.36), Camanducaia-Monte Verde (0.35), and Poços de Caldas-Bocaina de Minas (0.30), whereas it was lowest for the pairs Camanducaia-Poços de Caldas (0.07), Bocaina de Minas-Camanducaia (0.09), Bocaina de Minas-Monte Verde (0.09), and Poços de Caldas-Monte Verde (0.09). The species Cabralea canjerana, Lamanonia ternata, Myrcia splendens, Myrsine umbellata, and Prunus myrtifolia, were sampled at all study sites.

\section{Discussion}

Some of the richest families in our study, including Myrtaceae, Melastomataceae, Aquifoliaceae, and Symplocaceae, have been classified as typical of neotropical cloud forests (Webster 1995). In a study that investigated

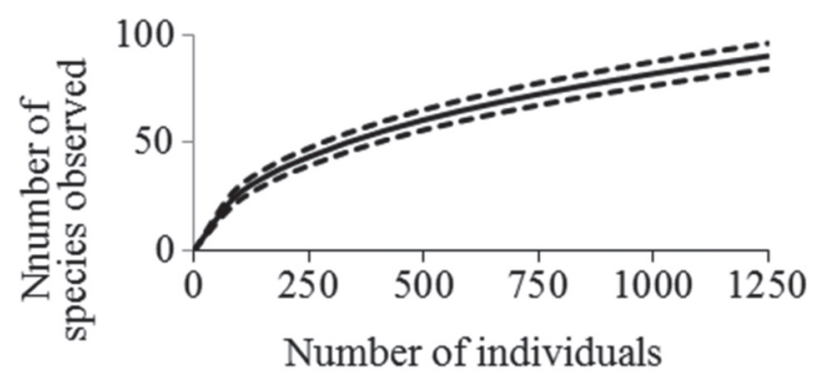

Figure 1. Tree species accumulation curve in an upper montane cloud forest at $1900 \mathrm{~m}$ elevation in the Serra da Mantiqueira Mountain Range, Itamonte County, in the state of Minas Gerais, Brazil. 
Floristic composition and structure of an upper montane cloud forest in the Serra da Mantiqueira Mountain Range of Brazil

Table 2. Texture and chemical analysis of soil samples from 15 plots $(10 \times 40 \mathrm{~m}$ each $)$ in an upper montane cloud forest at $1900 \mathrm{~m}$ elevation in the Serra da Mantiqueira Mountain Range, Itamonte County, in the state of Minas Gerais, Brazil.

\begin{tabular}{|c|c|c|c|c|c|c|c|c|c|c|c|c|c|c|c|c|c|}
\hline Plot & $\begin{array}{l}\text { Sand } \\
(\%)\end{array}$ & $\begin{array}{l}\text { Silt } \\
(\%)\end{array}$ & $\begin{array}{l}\text { Clay } \\
(\%)\end{array}$ & $\mathrm{pH}$ & $\begin{array}{c}\mathrm{P} \\
(\mathrm{mg} \times \\
\mathrm{dm}-3)\end{array}$ & $\begin{array}{c}\mathrm{K} \\
\mathrm{mg} \times \\
\mathrm{dm}-3)\end{array}$ & $\begin{array}{c}\mathrm{Ca} \\
(\mathrm{cmol} \times \\
\mathrm{dm}-3)\end{array}$ & $\begin{array}{c}\mathrm{Mg} \\
(\mathrm{cmol} \times \\
\mathrm{dm}-3)\end{array}$ & $\begin{array}{c}\mathrm{Al} \\
(\mathrm{cmol} \times \\
\mathrm{dm}-3)\end{array}$ & $\begin{array}{l}\mathrm{H}+\mathrm{Al} \\
(\mathrm{cmol} \times \\
\mathrm{dm}-3)\end{array}$ & $\begin{array}{c}\mathrm{SB} \\
(\mathrm{cmolc} \times \\
\mathrm{dm}-3)\end{array}$ & $\begin{array}{c}\mathrm{t} \\
(\mathrm{cmolc} \times \\
\mathrm{dm}-3)\end{array}$ & $\begin{array}{c}\mathrm{T} \\
(\mathrm{cmolc} \times \\
\mathrm{dm}-3)\end{array}$ & $\begin{array}{l}\mathrm{V} \\
(\%)\end{array}$ & $\begin{array}{l}\mathrm{m} \\
(\%)\end{array}$ & $\begin{array}{c}\mathrm{OM} \\
(\mathrm{dag} \times \mathrm{kg}-1)\end{array}$ & $\begin{array}{c}\text { P-rem } \\
(\mathrm{mg} \times \mathrm{L}-1)\end{array}$ \\
\hline P1 & 20 & 30 & 50 & 4.3 & 2.6 & 78 & 0.1 & 0.1 & 2.8 & 19 & 0.4 & 3.2 & 19.5 & 0.02 & 0.88 & 9.2 & 3.4 \\
\hline P2 & 20 & 47 & 30 & 4.4 & 3.1 & 67 & 0.1 & 0.1 & 2.7 & 17 & 0.4 & 3.1 & 17.5 & 0.02 & 0.88 & 10.0 & 3.8 \\
\hline P3 & 30 & 49 & 30 & 4.6 & 3.1 & 56 & 0.1 & 0.1 & 4.3 & 24 & 0.3 & 4.6 & 24.3 & 0.01 & 0.93 & 10.0 & 2.4 \\
\hline P4 & 40 & 36 & 20 & 4.6 & 3.1 & 45 & 0.1 & 0.1 & 3.1 & 17 & 0.3 & 3.4 & 17.4 & 0.02 & 0.91 & 10.3 & 2.6 \\
\hline P5 & 50 & 34 & 20 & 5.0 & 4.0 & 61 & 0.1 & 0.1 & 1.4 & 14 & 0.4 & 1.8 & 14.1 & 0.03 & 0.80 & 14.6 & 2.5 \\
\hline P6 & 30 & 31 & 40 & 5.0 & 2.0 & 80 & 0.1 & 0.1 & 2.1 & 15 & 0.4 & 2.5 & 15.7 & 0.03 & 0.84 & 8.7 & 11.0 \\
\hline P7 & 10 & 45 & 40 & 4.4 & 4.3 & 61 & 0.2 & 0.1 & 2.9 & 15 & 0.5 & 3.4 & 15.8 & 0.03 & 0.86 & 9.7 & 2.5 \\
\hline P8 & 20 & 42 & 40 & 4.4 & 4.3 & 76 & 0.1 & 0.1 & 3.0 & 19 & 0.4 & 3.4 & 19.5 & 0.02 & 0.88 & 11.5 & 1.9 \\
\hline P9 & 10 & 46 & 40 & 4.4 & 4.0 & 51 & 0.1 & 0.1 & 2.7 & 17 & 0.3 & 3.0 & 17.5 & 0.02 & 0.89 & 11.8 & 1.7 \\
\hline P10 & 10 & 38 & 50 & 4.5 & 3.1 & 64 & 0.1 & 0.1 & 3.2 & 19 & 0.4 & 3.6 & 19.5 & 0.02 & 0.90 & 10.0 & 2.3 \\
\hline P11 & 20 & 31 & 50 & 4.3 & 2.6 & 78 & 0.1 & 0.1 & 2.8 & 19 & 0.4 & 3.2 & 19.5 & 0.02 & 0.88 & 9.2 & 3.4 \\
\hline P12 & 20 & 30 & 50 & 4.4 & 3.1 & 67 & 0.1 & 0.1 & 2.7 & 17 & 0.4 & 3.1 & 17.5 & 0.02 & 0.88 & 10.0 & 3.8 \\
\hline P13 & 10 & 39 & 50 & 4.6 & 3.1 & 56 & 0.1 & 0.1 & 4.3 & 24 & 0.3 & 4.6 & 24.3 & 0.01 & 0.93 & 10.0 & 2.4 \\
\hline P14 & 20 & 35 & 40 & 4.6 & 3.1 & 45 & 0.1 & 0.1 & 3.1 & 17 & 0.3 & 3.4 & 17.4 & 0.02 & 0.91 & 10.3 & 2.6 \\
\hline P15 & 30 & 49 & 30 & 5.0 & 4.0 & 61 & 0.1 & 0.1 & 1.4 & 14 & 0.4 & 1.8 & 14.1 & 0.03 & 0.80 & 14.6 & 2.5 \\
\hline Mean & 23 & 39 & 39 & 4.6 & 3.3 & 63 & 0.1 & 0.1 & 2.8 & 18 & 0.4 & 3.2 & 18.2 & 0.0 & 0.9 & 10.7 & 3.3 \\
\hline SD & 8.9 & 6.1 & 8.4 & 0.2 & 0.5 & 9.1 & 0.0 & 0.0 & 0.5 & 2.3 & 0.0 & 0.5 & 2.3 & 0.0 & 0.0 & 1.3 & 1.2 \\
\hline
\end{tabular}

$\mathrm{V}$ - base saturation; $\mathrm{m}$ - aluminum saturation; $\mathrm{SB}$ - sum of bases; $\mathrm{t}$ - cation exchange capacity; $\mathrm{T}$ - cation exchange capacity at $\mathrm{pH}$ 7.0; $\mathrm{OM}$ - organic matter); $\mathrm{P}$ -rem - remaining phosphorus; SD - standard deviation.

Table 3. Comparison between an upper montane cloud forest at $1900 \mathrm{~m}$ elevation in the Serra da Mantiqueira Mountain Range, Itamonte County, in the state of Minas Gerais, Brazil (present study) and other cloud forests in the Serra da Mantiqueira.

\begin{tabular}{|c|c|c|c|c|c|c|c|}
\hline Site (Reference) & Elevation $(\mathrm{m})$ & $\mathrm{S}$ & $\mathrm{H}^{\prime}$ & $J^{\prime}$ & $\mathrm{D}$ & ADo & Species with the highest IV* \\
\hline \multirow{4}{*}{ Itamonte } & \multirow{6}{*}{$1890-1925$} & \multirow{6}{*}{89} & \multirow{6}{*}{3.49} & \multirow{7}{*}{0.78} & \multirow{6}{*}{2083} & \multirow{6}{*}{33.0} & Roupala rhombifolia \\
\hline & & & & & & & Myrsine umbellata \\
\hline & & & & & & & Pimenta pseudocaryophyllos \\
\hline & & & & & & & Myrceugenia miersiana \\
\hline \multirow[t]{2}{*}{ (Present study) } & & & & & & & Myrcia splendens \\
\hline & & & & & & & Prunus myrtifolia \\
\hline \multirow{3}{*}{ Monte Verde } & & & & & & & Pimenta pseudocaryophyllus \\
\hline & & & & & & & Roupala rhombifolia \\
\hline & $1820-1940$ & 64 & 3.28 & 0.78 & 3403 & 37.7 & $\begin{array}{l}\text { Drimys brasiliensis } \\
\text { Miconia cinerascens }\end{array}$ \\
\hline \multirow[t]{3}{*}{ (Meireles et al. 2008) } & & & & & & & Myrceugenia myrcioides \\
\hline & & & & & & & Myrceugenia brevipedicellata \\
\hline & & & & & & & Ocotea lancifolia \\
\hline \multirow[t]{2}{*}{ Camanducaia } & & & & & & & Cabralea canjerana \\
\hline & 1900 & 58 & 2.9 & 0.71 & 2001 & 48.1 & $\begin{array}{l}\text { Psychotria vellosiana } \\
\text { Myrcia splendens }\end{array}$ \\
\hline \multirow[t]{3}{*}{ (França \& Stehmann 2004) } & & & & & & & Drimys brasilienis \\
\hline & & & & & & & Myrsine umbellata \\
\hline & & & & & & & Vochysia magnifica \\
\hline \multirow[t]{2}{*}{ Bocaina de Minas } & & & & & & & Psychotria vellosiana \\
\hline & $1210-1360$ & 221 & 4.15 & 0.82 & 2475 & 33.3 & $\begin{array}{l}\text { Lamanonia ternata } \\
\text { Alchornea triplinervia }\end{array}$ \\
\hline \multirow[t]{3}{*}{ (Carvalho et al. 2005) } & & & & & & & Tibouchina pulchra \\
\hline & & & & & & & Casearia arborea \\
\hline & & & & & & & Alchornea triplinervia \\
\hline \multirow[t]{2}{*}{ Poços de Caldas } & & & & & & & Pera glabrata \\
\hline & $1200-1575$ & 156 & 4.26 & 0.56 & 1783 & 29.9 & $\begin{array}{l}\text { Calyptranthes widgreniana } \\
\text { Eugenia dodanaefolia }\end{array}$ \\
\hline \multirow[t]{2}{*}{ (Costa et al. 2011) } & & & & & & & Myrsine umbellata \\
\hline & & & & & & & Cyathea phalerata \\
\hline
\end{tabular}

S - number of species sampled; H' - Shannon diversity index; J' - Pielou's evenness index; D - density (number of individuals sampled); ADo - absolute dominance $\left(\mathrm{m}^{2} / \mathrm{ha}\right)$.

* Species are listed in decreasing order by IV (top six for each site). 
Patrícia Vieira Pompeu, Marco Aurélio Leite Fontes, Rubens Manoel dos Santos, Paulo Oswaldo Garcia,

Talita Alves Batista, Warley Augusto Caldas Carvalho and Ary Teixeira de Oliveira Filho

36 areas of montane forest in the Andes, Gentry (1995) reported that the three richest families between $1500 \mathrm{~m}$ and $2500 \mathrm{~m}$ in elevation were Lauraceae, Melastomataceae, and Rubiaceae. The families Myrtaceae, Aquifoliaceae, and Lauraceae have also been shown to be highly representative of the structure of upper montane cloud forests in the state of Paraná, Brazil (Scheer et al. 2011). In the upper montane cloud forest at Camanducaia, which, like our study area, is at an elevation of $1900 \mathrm{~m}$, França \& Stehmann (2004) found that the richest families were Myrtaceae, Solanaceae, Lauraceae, Rubiaceae, Symplocaceae, and Asteraceae. In another study conducted in the Serra da Mantiqueira, Meireles et al. (2008) found that, along an elevational gradient ranging from $1820 \mathrm{~m}$ to $1940 \mathrm{~m}$, the richest family was Myrtaceae, followed by Asteraceae, Lauraceae, Aquifoliaceae, Melastomataceae, Solanaceae, and Cunoniaceae. In the upper montane cloud forest at Bocaina de Minas, where the elevation ranges from $1210 \mathrm{~m}$ to $1360 \mathrm{~m}$, Carvalho et al. (2005) found Melastomataceae and Myrtaceae to be the richest families, followed by Fabaceae, Lauraceae, Annonaceae, Solanaceae, Euphorbiaceae, Asteraceae, Cyatheaceae, and Rubiaceae. Other authors have also identified Myrtaceae as the richest family in montane forests within the Atlantic Forest Biome of Brazil, including other forests in the Serra da Mantiqueira (Pereira et al. 2006; França \& Stehmann, 2004; Meirelles et al. 2008), as well as in other regions of the country, including the south (Scheer et al. 2011) and southeast (Oliveira-Filho \& Fontes 2000; Costa et al. 2011). Conversely, the family Fabaceae was not found in our study area, and its absence at higher elevations has also been noted in other studies (Oliveira-Filho \& Fontes, 2000; Gentry 1995; França \& Stehmann, 2004; Meireles et al. 2008; Scheer et al. 2011). In addition, certain ecological patterns observed in our study area, such as the presence of bamboos and epiphytes (e.g., bromeliads, mosses, and orchids) are also typical of tropical cloud forests (Stadtmüller 1987; Bruijnzeel \& Hamilton 2000; Bruijnzeel 2001; Scatena et al. 2010).

The genera Drimys, Ilex, Clethra, Meliosma, Cyathea, Miconia, Prunus, Roupala, and Rhamnus, all of which were sampled in our study, are considered diagnostic of neotropical cloud forests (Webster 1995). One of those genera (Miconia) has been listed as typical of montane forests in southeastern Brazil, as have the genera $\mathrm{Eu}$ genia, Ocotea, Myrcia, Mollinedia, Solanum, Tibouchina, Psychotria, Maytenus, Marlierea, Myrsine, Myrceugenia, Rudgea, and Symplocos (Oliveira-Filho \& Fontes 2000). At the Itamonte site, we sampled several of the species listed by Oliveira-Filho \& Fontes (2000) as indicator species of montane forests (semideciduous and ombrophilous forests) within the Atlantic Forest Biome: Byrsonima laxiflora, Calyptranthes clusiifolia, Clethra scabra, Daphnopsis fasciculata, Drimys brasiliensis, Heisteria silvianii, Maytenus salicifolia, Ouratea semiserrata, Pimenta pseudocaryophyl- lus, Siphoneugena widgreniana, and Symplocos celastrinea. Some of the species found to be common to all of the cloud forests compared in the present study-Cabralea canjerana, Lamanonia ternata, Myrcia splendens, Myrsine umbellata, and Prunus myrtifolia - were not mentioned by Bertoncello et al. (2011) or Oliveira-Filho \& Fontes (2000) as being preferred species of cloud forests. However, our findings suggest that these species are in fact associated with such forests. The fact that Myrsine umbellata had high importance values in three of the cloud forests evaluated demonstrates the importance of this species not only floristically but also in the structural organization of these communities. In a study employing two-way indicator species analysis, Bertoncello et al. (2011) identified the species Blepharocalyx salicifolius, Citronella paniculata, and Rhamnus sphaerosperma (all sampled in the present study) as preferred species for occurrence in cloud forests within the Atlantic Forest Biome in southern and southeastern Brazil, as well as identifying Drimys brasiliensis as indicative of cloud forests. The authors demonstrated that cloud forests form a distinct floristic and phytogeographic unit.

The CCA failed to detect any gradients of vegetation or soil, indicating the homogeneity of the community studied, in terms of those variables. However, Meirelles et al. (2008) found variations in forest structure and high species turnover along a short elevational gradient (1820-1940 m) in Monte Verde. According to the authors, that is due to the fact that the plots located at the lower elevations were close to a stream, whereas those located at the higher elevations were more exposed to the wind. In the forest communities of Bocaina de Minas and Poços de Caldas, both of which are at the lower elevations of the Serra da Mantiqueira, Carvalho et al. (2005) and Costa et al. (2011), respectively, also found low species turnover, even when the plots were distributed along a soil gradient. Therefore, we can classify the Serra da Mantiqueira as environmentally heterogeneous, which makes it difficult to standardize the behavior of its tree community.

The forest in Itamonte is among the richest and most diverse cloud forests in the Serra da Mantiqueira, as previously demonstrated by Scheer et al. (2011). However, at Camanducaia-under like environmental conditions (at an elevation of $1900 \mathrm{~m}$, over comparable soil, with a similar microclimate, and without an elevational gradient)_França \& Stehmann (2004) reported low diversity and species richness, despite having evaluated a larger sample (1378 trees in a 0.75 -ha area). Such a decrease in diversity at the upper elevations has been reported for other tropical mountain ranges (Gentry, 1995). Furthermore, the heterogeneity in the spatial organization in abundance and basal area of cloud forests communities evidence the importance of conservation of distinct remnant in function of structural variability.

Scheer et al. (2011) used cluster analysis to determine the importance of tree species in upper montane cloud forests 
of the Serra do Mar Mountain Range in the state of Paraná, the Aparados da Serra Geral Mountain Range in the state of Santa Catarina, and the Serra da Mantiqueira. The authors recognized the vegetation of the Serra da Mantiqueira as a distinct floristic group. However, our study showed that the tree communities of the Serra da Mantiqueira are characterized by high vegetation heterogeneity, indicating the need for further studies in the region.

The tree community in Itamonte is characterized by high species richness and diversity. The structural and floristic heterogeneity among the various communities compared makes it is difficult to categorize the cloud forests of the Serra da Mantiqueira by floristic or structural patterns. Therefore, there is a need for larger, more detailed floristic studies of these areas. Our findings also underscore the need for better conservation of these ecosystems that are unique and irreplaceable, especially at their upper elevations.

\section{Acknowledgments}

We are grateful to the Alto-Montana da Serra Fina Institute (the host institution), for the logistical support provided, as well as to Prof. Marcos Sobral, of the Universidade Federal de São João del-Rei (UFSJ, Federal University at São João del-Rei), and Prof. Leonardo Dias Meireles, of the Universidade de São Paulo (USP, University of São Paulo), for their valuable contributions. This study received financial support from the Boticário Group Foundation for Nature Preservation (funding for the project "The interception of fog by Atlantic Upper Montane Cloud Forest: a study of the correlation between vegetation, meteorological factors, and cloud effect along an elevational gradient in the Serra da Mantiqueira mountain range") and from the Brazilian Conselho Nacional de Desenvolvimento Científico e Tecnológico (CNPq, National Council for Scientific and Technological Development; scholarship to PVP).

\section{References}

Angiosperm Phylogeny Group - APG III. 2009. An update of the Angiosperm Phylogeny Group classification for the orders and families of flowering plants: APG III. Botanical Journal of the Linnean Society 161: 105-21.

Bertoncello, R.; Yamamoto, K.; Meireles, L.D. \& Shepherd, G.J. 2011. A phytogeographic analysis of cloud forests and other forest subtypes amidst the Atlantic forests in south and southeast Brazil. Biodiversity and Conservation 20: 3413-3433.

Brower, J.E. \& Zar, J.H. 1984. Field and laboratory methods for general ecology. Dubuque, W.M.C. Brow.

Bruijnzeel, L.A. \& Hamilton, L.S. 2000. Decision Time for Cloud Forests. IHP Humid Tropics Programme Series n. 13, IHP-UNESCO, Paris, IUCN-NL, Amsterdam and WWF International, Gland.

Bruijnzeel, L.A. 2001. Hydrology of tropical montane cloud forests: A Reassessment. Land Use and Water Resources Research 1(1): 1-18.

Bruijnzeel, L.A.; Kappelle, M.; Mulligan, M. \& Scatena, F.N. 2010. Tropical montane cloud forests: state of knowledge and sustainability perspectives in a changing world. Pp.691-740. In: Bruijnzeel, L.A., Scatena F.N. \& Hamilton, L.S. (eds.). Tropical Montane Cloud Forests. Sci- ence for Conservation and Management. Cambridge, Cambridge University Press.

Carvalho, D.A.; Oliveira-Filho, A.T.; Van Den Berg, E.; Fontes, M.A.L.; Vilela, E.A.; Marques, J.J.G.S.M \& Carvalho, W.A.C. 2005. Variações florísticas e estruturais do componente arbóreo de uma floresta ombrófila alto-montana às margens do rio Grande, Bocaina de Minas, MG, Brasil. Acta Botanica Brasilica 19(1): 91-109.

Costa, M.P.; Pereira, J.A.A; Melo, P.H.A.; Pífano, D.S.; Pellicciottii, A.S; Pompeu, P.V. \& Silva, R.A. 2011. Estrutura e diversidade da comunidade arbórea de uma floresta superomontana, no planalto de Poços de Caldas (MG). Ciência Florestal 21(4): 711-725.

EMBRAPA. 1997. Manual de métodos de análise de solo. Rio de Janeiro, Empresa Brasileira de Pesquisa Agropecuária, Centro Nacional de Pesquisa de Solos.

França G.S. \& Stehmann, J.R. 2004. Composição florística e estrutura do componente arbóreo de uma floresta altimontana no município de Camanducaia, Minas Gerais, Brasil. Revista Brasileira de Botânica 27(1): 19-30.

Gentry, A.H. 1995. Patterns of diversity and floristic composition in neotropical montane forests. Pp. 103-126. In: Churchill, S. P.; Balslev, H.; Forero, E. \& Luteyn, J. L. (Eds.). Biodiversity and Conservation of Neotropical Montane Forests. In: Neotropical montane forest biodiversity and conservation symposium, 1993, New York. Proceedings... New York, The New York Botanical Garden.

IBGE. 2012. INSTITUTO BRASILEIRO DE GEOGRAFIA E ESTATÍSTICA. Manual técnico da vegetação brasileira. Disponível em: ftp://geoftp.ibge.gov.br/documentos/recursos_naturais/manuais_tecnicos/manual_tecnico_vegetacao_brasileira.pdf. Acessado em 25 jan. 2013.

Le-Saout, S.; Hoffmann, M.; Shi, Y.; Hughes, A.; Bernard, C.; Brooks, T. M.; Bertzky, B.; Butchart, S.H.M.; Stuart, S.N.; Badman, T. \& Rodrigues, A.S.L. 2013. Protected Areas and Effective Biodiversity Conservation. Science 342: 803-805.

Mccune, B. \& Mefford, M.J. Multivariate Analysis of Ecological Data: PC-ORD version 5.10. Oregon, MjM Software.

Meireles, L.D.; Shepherd, G.J. \& Kinoshita, L.S. 2008. Variações na composição florística e na estrutura fitossociológica de uma floresta ombrófila densa alto-montana na Serra da Mantiqueira, Monte Verde, MG. Revista Brasileira de Botânica 31(4): 559-574.

Muller-Dombois, D. \& Ellenberg, H. 1974. Aims and methods of vegetation ecology. New York, John Wiley \& Sons.

Oliveira-Filho, A.T. \& Fontes, M.A.L. 2000. Patterns of Floristic Differentiation among Atlantic Forests in Southeastern Brazil and the Influence of Climate. Biotropica 32(4): 793-810.

Oliveira-Filho, A.T. 2009. Classificação das fitofisionomias da América do Sul cisandina tropical e subtropical: proposta de um novo sistema - prático e flexível - ou uma injeção a mais de caos? Rodriguésia 60 (2): 237-258.

Pane, E. \& Pereira, S.Y. 2005. As fontes em Itamonte, sul de Minas Gerais - uma contribuição para o entendimento das relações entre água superficial e subterrânea. Águas Subterrâneas 19 (1): 1-14.

Pereira, I.M.; Oliveira-Filho, A.T.; Botelho, S.A.; Carvalho, W.A.C.; Fontes, M.A.L.; Schiavini, I. \& Silva, A.F. 2006. Composição florística do compartimento arbóreo de cinco remanescentes florestais do maciço do Itatiaia, Minas Gerais e Rio de Janeiro. Rodriguésia 57(1): 103-126.

Sá Júnior, A.; Gonsaga, L.C.; Silva, F.F.; Alves, M.C. 2012. Application of the Köppen classification for climatic zoning in the state of Minas Gerais, Brazil. Theoretical and Applied Climatology 108: 1-7.

Scatena, F.N.; Bruijnzeel, L.A.; Bubb, P.; Das, S. 2010. Setting the stage. Pp. 3-13. In: Bruijnzeel, L.A., Scatena F.N. \& Hamilton, L.S. (eds.). Tropical Montane Cloud Forests. Science for Conservation and Management. Cambridge, Cambridge University Press.

Scheer, M.B.; Mocochinski, A.Y. \& Roderjan, C.V. 2011. Estrutura arbórea da Floresta Ombrófila Densa Altomontana de serras do Sul do Brasil. Acta Botanica Brasilica 25 (4): 735-750.

Stadtmüller, T. 1987. Cloud Forests in the humid tropics: a bibliographic review. Tokyo and Turrialba. The United Nations University; Centro Agronomico Tropical de Investigacion y Ensenanza. 
Patrícia Vieira Pompeu, Marco Aurélio Leite Fontes, Rubens Manoel dos Santos, Paulo Oswaldo Garcia, Talita Alves Batista, Warley Augusto Caldas Carvalho and Ary Teixeira de Oliveira Filho

ter Braak, C.J.F. 1995. Ordination. Pp. 91-173. In: Jongman, R.H.G.; ter Braak, C.J.F. \& van Tongeren, O.F.R. (Eds.). Data analysis in community and landscape ecology. Cambridge, Cambridge University Press.

Thiers, B. [continuously updated]. Index Herbariorum: a global directory of public herbaria and associated staff. New York Botanical Garden's Virtual Herbarium. Website http://sweetgum.nybg.org/ih/. [accessed on October, 15, 2013].
Webster, G. L. 1995. The panorama of Neotropical Cloud Forests. Pp 5377. In: Churchill, S. P.; Balslev, H.; Forero, E. \& Luteyn, J. L. (Eds.). Biodiversity and Conservation of Neotropical Montane Forests. In: Neotropical montane forest biodiversity and conservation symposium, 1993, New York. Proceedings... New York, The New York Botanical Garden.

Zar, J.H. 2010. Biostatistical analysis. New Jersey, Prentice Hall. 